\title{
Nachruf auf Wolfgang Dihlmann
}

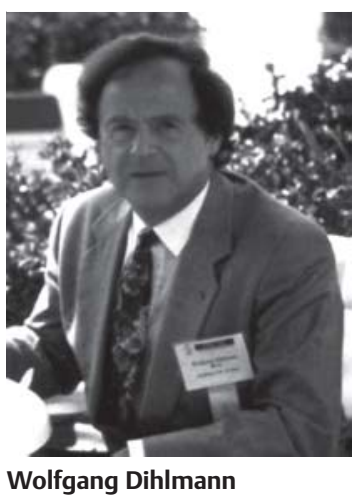

Wolfgang Dihlmann
Wolfgang Dihlmann war in seiner Persönlichkeit und als Radiologe in vieler Hinsicht außergewöhnlich. Die Größe seines Lebenswerks erschließt sich dem Leser daher durchaus nicht immer unmittelbar. Sein Opus magnum, die 2011 in der 4. Auflage erschienenen „Gelenke und Wirbelverbindungen“" stellen mit ihren 1002 Seiten den wahrscheinlich letzten und erfolgreichen Versuch dar, das derzeitige Wissen der muskuloskelettalen Radiologie in einer anspruchsvollen Form zusammenzufassen. Den kernspintomografischen Part hat Dihlmann in der 4. Auflage seinem Koautor Axel Stäbler anvertraut. Die Methode wird damit zum nahtlosen Bestandteil von Dihlmanns Gesamtkonzept.

Das Außergewöhnliche zeigt sich bei dem 1928 in Stettin geborenen Wolfgang Dihlmann auch in seiner Lebensgeschichte. Mit 15 1/2 Jahren als Flakhelfer eingezogen, gerät er als verwundeter Grenadier in sowjetische Kriegsgefangenschaft. Ab 1946 kann er in Greifswald, Halle/Saale und Jena studieren. Nach Stationen in Magdeburg, Rostock und als Oberarzt an der Charité wird er 1960 Leiter der Röntgenabteilung der Aachener Rheumaklinik Landesbad. Er wechselt dann als Oberarzt zu W. Frik in die Abteilung Radiologie der RWTH Aachen, habilitiert dort und wird 1971 außerordentlicher Professor für Klinische Radiologie. Von 1974-1993 leitet Dihlmann als Chefarzt das Röntgeninstitut des Allgemeinen Krankenhauses Hamburg-Barmbek. Von seinen zahlreichen nationalen und internationalen Auszeichnungen seien hier nur genannt:

- 1970: Hermann-Holthusen-Ring der Deutschen Röntgengesellschaft

- 1987: Präsident des 68. Deutschen Röntgenkongresses in Hamburg

- 1987 erhielt sein „Röntgenatlas rheumatischer Erkrankungen“ in der englischen Übersetzung den „Pulitzerpreis“ der amerikanischen Medizinjournalisten

- 1999: Ehrenmitglied der Europäischen Gesellschaft für Skelettradiologie

- 2002: Ehrenmitglied der Deutschen Gesellschaft für Rheumatologie

- 2005: Goldmedaille des Europäischen Kongresses für Radiologie (ECR) und der European Association of Radiology (EAR)

Neben über 300 Vorträgen und über 300 wissenschaftlichen Publikationen, sowie 7 Fachbüchern war Dihlmann auch Mitbegründer der Zeitschrift „Aktuelle Rheumatologie“ und Mitherausgeber der „Zeitschrift für Rheumatologie“ sowie des „Schinz“. In der 1995 erschienen „Gelenkshilouette" hat Dihlmann in seiner, so klugen wie humorvollen, Art und Weise die streng naturwissenschaftlichen, die wahrnehmungsphysiologischen und die erkenntnistheoretischen Grundlagen der Radiologie skizziert und miteinander verbunden. Eine Reihe von Entitäten und Röntgensymptomatologien wurden von Dihlmann erstmals beschrieben und können hier nur sehr unvollständig aufgeführt werden: Die Sacroiliitis circumscripta, die unterschiedlichen Spondylodiscitiden bei der Spondylitis ancylosans, die Hyperostosis triangularis ilii, die Spondylosclerosis hemispherica sowie das Aquirierte Hyperostose Syndrom.

Im Dialog war Dihlmann stets selbstkritisch, humorvoll und immer offen für das bessere $\mathrm{Ar}$ gument. Er war als lernender Lehrer Vorbild in seinem Fach, insbesondere aber auch für seine Mitarbeiter und Schüler.

Am 3. Oktober 2013 ist Prof. Wolfgang Dihlmann in München verstorben.

Lutz Hering

Gerwin Lingg

Hamburg
Bibliografie

DOI http://dx.doi.org/10.1055/

s-0033-1356143

Fortschr Röntgenstr 2013; 185:

1137 @ Georg Thieme Verlag KC

Stuttgart · New York .

ISSN 1438-9029

Korrespondenzadresse

Dr. med. Gerwin Lingg

ehem. Chefarzt am Accura-

Rheumazentrum-Rheinland-

Pfalz

Mainz Bad Kreuznach

Kaiser-Wilhelm-Straße 9-11

Privat Adresse:

Nelli-Schmithals-Str. 52

D-55543 Bad Kreuznach

gerwin-lingg@web.de 